\title{
Le voyage des écrivains polonais en Europe
}

Anne-Marie Monluçon et Anna Saignes

\section{OpenEdition}

Journals

Édition électronique

URL : https://journals.openedition.org/recherchestravaux/843

DOI : 10.4000/recherchestravaux.843

ISSN : 1969-6434

Éditeur

UGA Éditions/Université Grenoble Alpes

Édition imprimée

Date de publication : 1 décembre 2016

Pagination : 5-16

ISBN : 978-2-84310-338-4

ISSN : 0151-1874

\section{Référence électronique}

Anne-Marie Monluçon et Anna Saignes, "Le voyage des écrivains polonais en Europe ", Recherches \& Travaux [En ligne], 89 | 2016, mis en ligne le 12 janvier 2017, consulté le 29 octobre 2021. URL : http:// journals.openedition.org/recherchestravaux/843; DOI : https://doi.org/10.4000/recherchestravaux. 843 
Anne-Marie Monluçon, Anna Saignes

Univ. Grenoble Alpes, UMR 5316 Litt\&Arts

\section{Le voyage des écrivains polonais en Europe}

On partait pour une guerre étrangère, on se sauvait devant les armées, on fuyait la misère et la faim.

Voilà pourquoi l'Europe centrale n'a jamais eu ses grands voyageurs. Elle était occupée à voyager dans ses propres limites. Partir en voyage par curiosité? Pareille pensée ne peut venir à l'esprit que si nous sommes persuadés que rien n'arrivera à notre maison, si nous savons sans l'ombre d'un doute qu'à notre retour notre maison sera toujours là ${ }^{\mathrm{I}}$.

Le titre du colloque que prolonge le présent volume, "La Pologne, citoyenne du monde : regards croisés sur les écrivains voyageurs polonais $\left(\mathrm{XIX}^{\mathrm{e}}-\right.$ $\mathrm{XXI}^{\mathrm{e}}$ siècles $)^{2}$ " peut surprendre au regard des clichés associés à ce pays européen et à sa récente actualité politique. Pourtant, la simple consultation des rayons de librairie ou des catalogues d'éditeurs révèle un paradoxe. Parmi les trop rares

I. A. Stasiuk (avec I. Andrukhovych), Mon Europe, trad. M. Malanchuk, M. Laurent, Montricher, Noir sur Blanc, 2004, p. I09; "Wyjeżdżato się na cudzq wojnę, uciekato przed armiami, umykato przed nędza i gtodem. Dlatego Europa Środkowa nigdy nie wydata wielkich podróżników. Zajęta byta podróżowaniem w swoim wtasnym wnętrzu. Wybrać się $w$ drogę $z$ ciekawości? Taka myśl może przyjść do gtowy komus, kto jest pewien nienaruszalności wtasnego domu, komus, kto nie zastanawia się nad tym, czy po powrocie jego wtasny dom będzie stat na tym samym miejscu." (A. Stasiuk, Moja Europa, Wolowiec, Czarne, 2000, p. II2-II3.)

2. Ce colloque (organisé à l'université Stendhal-Grenoble 3, du 2I au 23 octobre 2015) a pu se tenir grâce au soutien du ministère de l'Enseignement supérieur et de la Recherche, de l'université Stendhal - Grenoble 3, de l'UMR 5316 Litt\&arts, de l'EA Ilcea4, de la fondation Jan Michalski, du consulat général de Pologne de Lyon, de Grenoble-Alpes Métropole, de l'Institut polonais et de l'Instytut Książki [Institut du livre] de Cracovie. Le colloque ainsi que ce volume se rattachent à l'axe structurant «Littératures et savoir» de l'UMR 5316 Litt\&arts. 
livres traduits du polonais, la Pologne, bien que souvent perçue comme nationaliste - et connaissant, actuellement, un repli identitaire - exporte particulièrement bien les œuvres de ses voyageurs et reporters. À l'heure où l'on proclame partout la fin du voyage, faut-il attribuer cette vitalité du récit de voyage polonais à une histoire propre aux anciens pays du «bloc de l'Est»? Les habitants de l'Europe centrale ont pendant longtemps été limités dans leur liberté de voyager. Les choses changent en 1989 seulement, même si l'effondrement du Rideau de fer ne règle pas la question financière : pour beaucoup de Polonais les voyages demeurent un luxe. L'histoire de cette partie de l'Europe ne serait pas encore tout à fait synchronisée avec la nôtre? La question est plus complexe, car, d'un côté, la fin du voyage n'empêche pas la littérature des voyageurs de se porter très bien à l'Ouest, et, de l'autre, la tradition du voyage et du reportage en Pologne est bien antérieure à la fin du régime communiste.

Ce numéro de Recherches \& Travaux est consacré à l'évolution d'une tradition ancienne, et fondamentalement européenne, celle des voyages de proximité, en Europe. Il sera prochainement complété par un volet consacré aux voyages lointains des écrivains polonais, qui paraittra dans la revue Slovo (Inalco). Cette réflexion s'inscrit dans le prolongement de travaux publiés antérieurement par Maria Delaperrière et Franciszek Ziejka³ ou par Małgorzata Smorag-Goldberg et Marek Tomaszewski ${ }^{4}$ Elle poursuit, d'autre part, le travail entamé dans le numéro de Recherches \& Travaux intitulé La Chute de l'URSS : une fin d'empire', ainsi que celui mené au sein de notre centre de recherche sur Le Voyage politique des écrivains et des cinéastes au XXe siècle ${ }^{6}$.

Le lecteur trouvera dans ce volume des articles consacrés à des auteurs connus, mais dont les traductions sont parfois épuisées, ainsi que plusieurs contributions visant à faire découvrir des écrivains encore jamais traduits en français, voire peu connus dans leur propre pays, comme Maria Komornicka, dont la redécouverte est récente, ou Henry (Khaim) Shoshkes. D’autre part,

3. M. Delaperrière (dir.), Joseph Conrad, un Polonais aux confins de l'Occident, Paris, Institut d'études slaves, 2009; M. Delaperrière, F. Ziejka (dir.), La Pologne multiculturelle, Paris, Institut d'études slaves, 20 IO.

4. M. Smorag-Goldberg, M. Tomaszewski (dir.), Mémoire(s) des lieux dans la prose centreeuropéenne après 1989, Montricher, Noir sur Blanc, 2013.

5. A.-M. Monluçon, A. Saignes, A. Salha (dir.), Recherches \& Travaux, no 80, La Chute de l'URSS : une fin d'empire, $20 \mathrm{I2}$.

6. Colloque organisé conjointement dans les universités Stendhal-Grenoble 3 et Louis Lumière Lyon 2, les 8 et 9 mars 20I2, par M. Boyer-Weinmann, D. Carlat, D. Lançon, D. Coureau (à paraître).

7. L'anthologie du reportage polonais dirigée par M. Szczygieł et publiée récemment en Pologne n'est malheureusement accessible qu'en polonais et permet de mesurer les manques parmi les traductions françaises (M. Szczygieł [dir.], IOO/XX. Antologia polskiego reportażu 
les articles étudient la littérature du voyage dans ses formes les plus variées. Le clivage entre récit de voyage et reportage, brouillé par l'expression «reportage littéraire", forgée par la critique polonaise, apparaît ici dans toute sa complexité. Mais le voyage peut aussi se raconter dans un poème ou dans un essai, dans un récit autobiographique, une nouvelle ou un roman. Cette approche transgénérique est une originalité de notre volume.

Les études s'ordonnent en trois étapes. Le pôle de la tradition est représenté par le voyage en Europe de l'Ouest. Bien que la plus éloignée géographiquement, celle-ci est ressentie comme très proche culturellement, du moins par la génération des écrivains polonais formés aux humanités classiques, avant 1939. Leur destination est l'Italie, la Grèce, la France, à l'exception de Maria Komornicka qui explore, à la toute fin du XIx ${ }^{e}$ siècle, le campus de Cambridge, haut lieu des humanités classiques, il est vrai. Depuis 1989, les voyages se réorientent vers l'Europe centrale : ils explorent des régions plus proches de la Pologne, géographiquement, linguistiquement (régions de langue slave, à l'exception de la Hongrie) et géopolitiquement (anciens pays du bloc communiste). Lorsqu'Andrzej Stasiuk résume ses pérégrinations en 2000, dans un essai intitulé Mon Europe, il décrit sur la carte un cercle dont le centre est son village d'adoption, Wołowiec, situé dans les Beskides, montagnes du sud-est de la Pologne :

Le trait passe plus ou moins par Brest-Litovsk, Rivne, Tchernivtsi, Cluj-Napoka, Arad, Szeged, Budapest, Zilina, Katowice, Czestochowa, pour se terminer là où il commence et donc à Varsovie. Au centre se trouve un morceau de Biélorussie, une surface non négligeable d'Ukraine, des espaces signifiants de dimensions équivalentes de Roumanie et de Hongrie, la Slovaquie dans son entier et une parcelle de la Tchéquie. Et, bien évidemment, un tiers de ma patrie, la Pologne ${ }^{8}$.

Dans le troisième volet de ce volume, les articles consacrés au voyage en Pologne permettent d'observer que le voyage peut aussi être une manière de découvrir sa propre identité, c'est-à-dire d'acquérir une distance par rapport à celle-ci, mais aussi de reconnaître l'altérité qui nous habite.

Quelle serait la spécificité du récit de voyage polonais? Trois perspectives permettent de la mesurer : le contexte sociopolitique, la question du rapport à l'altérité et, enfin, la réflexion sur les formes de ces écrits.

XX wieku [Anthologie du reportage polonais au XXe siècle], vol. I-3, Wolowiec, Éditions Czarne, 2014-2015.)

8. Mon Europe, ouvr. cité, p. 84; "Linia biegnie mniej więcej przez Brześć, Równe, Czerniowce, Cluz-Napoka, Arad, Szeged, Budapeszt, Żylinę, Katowice, Częstochowe i kończy się tam, gdzie zaczyna, czyli w Warszawie. Wewnatrz jest kawatek Biatorusi, catkiem sporo Ukrainy, przyzwoite i porównywalne przestrzenie Rumunii i Wegier, prawie cata Stowacja i skrawek Czech. No i jakaś jedna trzecia Ojczyzny.» (Moja Europa, ouvr. cité, p. 86.) 


\section{L'évolution du contexte sociopolitique des voyages}

Jusqu'en 1956 le voyage en Europe de l'Ouest mène à ce que les intellectuels polonais considèrent comme le berceau de la civilisation européenne. Le voyage en Italie de la comtesse Potocka en est ici l'archétype. Elle reste dans l'entre-soi des aristocrates européens, bonapartistes de préférence. Du début du XIX ${ }^{\mathrm{e}}$ siècle à 1939, la Pologne connaît cependant plusieurs mutations politiques majeures : rayée de la carte en 1795 à la suite des trois partages, elle renaît comme État en 1918; la modernisation socioéconomique donne naissance à la presse et au reportage qui peut, dès lors, concurrencer le traditionnel récit de voyage. Une première rupture sociologique est amorcée dès la fin du XIX $^{e}$ siècle avec l'irruption dans le récit viatique de l'altérité sociale, aussi bien du côté des voyageurs que des objets que ces derniers choisissent. En d'autres termes, c'est le moment d'une première démocratisation de la sociologie du voyage. Władysław Reymont, issu d'une famille modeste (quoique cultivée), connu essentiellement pour ses romans ${ }^{9}$ et récompensé par le prix Nobel de littérature en 1924, est aussi l'auteur d'un texte destiné à la presse, intitulé Pielgrzymka do Jasnej Góry : wrażenia i notatki [Pèlerinage à Jasna Góra : impressions et notes], qui peut être considéré comme le premier reportage polonais au sens moderne du terme. Dans l'entre-deux-guerres, Khaim Shoskhes, citadin cultivé, part à la découverte des Juifs habitant les shtetlech des provinces reculées de Pologne. Il raconte ses voyages dans des textes destinés également à la presse.

Les années 1939-1945 sont celles des déplacements forcés, de la déportation, de la captivité, des opérations militaires. Il est impossible de considérer ces mouvements comme des voyages d'agrément, ni même de découverte de l'altérité. Tous ces voyageurs-par-la-force-des-choses ont néanmoins laissé des témoignages. Le journal d'Andrzej Bobkowski, En guerre et en paix [Szkice piórkiem], en constitue un exemple passionnant : cet ingénieur polonais, bloqué en France par le déclenchement de la guerre en septembre 1939, porte un regard de voyageur sur le pays qu'il traverse à vélo, tout en envisageant de manière très lucide la situation internationale.

Durant la période communiste, de 1945 à 1989 , il est très difficile de circuler en Pologne, même pour se rendre dans les "pays frères». Le dégel de 1956 apporte un relatif assouplissement dont quatre auteurs témoignent : Zbigniew Herbert, Stanisław Dygat, Sławomir Mrożek, Tadeusz Różewicz. Ils se dirigent vers l'Italie, effectuant un pèlerinage aux sources de la culture, qui prend pourtant une signification singulière dans son contexte politique : l'enjeu est,

9. La Terre promise [Ziemia obiecana], I897-I998; Les Paysans [Chtopi], I904-1909. 
pour eux, de recoudre la culture, de retrouver une unité brisée par le stalinisme, une tradition stratifiée, reflétant aussi bien les croyances des hommes que leurs compétences, leur art, leurs connaissances pratiques et théoriques sur le monde, une organisation sociale, un véritable "art de vivre».

La date de 1989 constitue, sans surprise, un tournant majeur, après cinquante ans d'enfermement. L'intégration européenne, en 2004, marque une étape supplémentaire. Il est désormais beaucoup plus facile de circuler. Andrzej Stasiuk refuse cependant de céder à l'euphorie en soulignant que des obstacles à la circulation demeurent, d'ordre économique ou socioculturel. Voici comment il résume sa conversation avec un ostalgique roumain, dans Sur la route de Babadag :

Il disait [...] que du temps de Ceauscescu, c'était mieux, qu’alors c'était la justice car c'était l'égalité, il y avait du travail et les rues étaient propres. [...]. Je ne disais donc plus rien car quaurais-je pu dire, une fois arrivé là, près de cette clôture, moi, manifestation flagrante de l'inégalité [...] ? [...] je sentais qu'en réalité, on n'en avait rien à foutre ici de ma liberté de venir et de repartir, qu' elle ne valait rien ${ }^{10}$.

Pourtant la fin du régime communiste ouvre bien la voie à un phénomène de massification des voyages. Désormais, les lecteurs sont aussi des voyageurs. En parallèle avec l'essor des voyages, on voit se développer une forme spécifique du récit de voyage : Maciej Kalarus, auteur d'une thèse intitulée Podróż ograbiona : polska literatura podróżnicza po I989 roku ${ }^{\mathrm{II}}$ [Le Voyage pillé : la littérature de voyage polonaise après 1989], comptabilise plus de trois cents titres, publiés depuis 2003 en Pologne. Les destinations sont désormais multiples, mais on notera parmi celles-ci un tropisme marqué vers l'Europe centrale, les Balkans, voire la Russie. Certains écrivains-voyageurs opèrent une ré-orient-tation de leurs pérégrinations, notamment vers l'ancien Est communiste. C'est le cas d'Andrzej Stasiuk, de Mariusz Szczygieł et de Krzysztof Varga. Devenu accessible, l'Ouest a certainement perdu de son attractivité.

La vitalité de la littérature du voyage signifie-t-elle que le thème est désormais devenu un argument de vente? Serait-ce parce que voyager n'est qu'une manière d'obéir à l'impératif de mouvement perpétuel, propre à notre époque, ainsi que le perçoit le sociologue Zygmunt Bauman? Les œuvres de Joanna Bator,

Io. A. Stasiuk, Sur la route de Babadag, trad. M. Maliszewska, Paris, Christian Bourgois, coll. "Littérature étrangère», 2007, p. 27-28; "Mówit [...] że kiedy rządzit Ceaucescu, byto lepiej, że wtedy byta sprawiedliwość, ponieważ byta równość, byta praca i porzadek na ulicah. [...] No więc nie mówitem już nic, bo cóż mogtem powiedzieć, skoro zjawitem się tutaj, przy tym ptocie, jako widomy znak nierówności [...]? [...] a ja czutem, że moja wolność przyjazdu i odjazdu gówno tutaj naprawdę znaczy i nic nie jest warta.» (A. Stasiuk, Jadąc do Babadag, Wolowiec, Éditions Czarne, 2004, p. 23-24.)

II. La thèse à été soutenue en 2013 à l'université de Katowice. 
d'Olga Tokarczuk et d'Andrzej Stasiuk font clairement écho à cette idée. Quoi qu'il en soit, il est révélateur que la majorité des écrivains voyageurs contemporains, en Pologne, sont des journalistes. Plusieurs d'entre eux ont commencé leur carrière dans le quotidien Gazeta Wyborcza [Journal des élections] $]^{12}$ fondé en mai 1989, au moment des premières élections libres : Jacek Hugo-Bader, Andrzej Stasiuk, Mariusz Szczygieł, Wojciech Tochman, Krzysztof Varga et Mariusz Wilk. Tous, à l'exception de Mariusz Wilk, publient leurs livres, souvent tirés de leurs articles et chroniques, aux Éditions Czarne [Noir], fondées en 1996 et dirigées par Andrzej Stasiuk et son épouse, Monika Sznajderman. Un grand nombre de ces textes sont traduits et diffusés par les Éditions Noir sur Blanc, spécialisées dans la publication des littératures de l'Est. Est-ce à dire que tous leurs livres relèvent du reportage et que le récit de voyage au sens traditionnel tend à disparaître? Est-ce à l'inverse l'indice que le journalisme est toujours un pis-aller et l'une des rares sources de revenus réguliers possibles pour les écrivains? Pour compléter le tableau social et institutionnel, notons que Paweł Goźliński, Mariusz Szczygieł et Wojciech Tochman ont fondé, en 2009 à Varsovie, un Instytut Reportażu [Institut du reportage], qui se donne pour mission de promouvoir le reportage polonais en diffusant de l'information sur son actualité, en organisant des manifestations, en proposant une formation et en publiant des textes, inédits ou méconnus. L'institut dispose également d'une librairie spécialisée dans le reportage et la littérature liée au voyage, en plein centre de Varsovie.

\section{Le proche, l'autre et le même}

Comment se passe la confrontation entre mythe et réalité? La comtesse Potocka résiste, refuse tout ce qui contredit son mythe italien, consistant à ne voir l'Italie que comme patrie des arts. Cette vision traditionnelle perdure étrangement chez certains voyageurs du $\mathrm{xx}^{\mathrm{e}}$ siècle. Czesław Miłosz intitule Rodzinna Europa [L'Europe familière $\left.{ }^{13}\right]$ un ouvrage dans lequel il s'efforce d'expliquer aux

I2. D'autres, ou les mêmes, publient également dans les hebdomadaires Polityka (de centregauche) ou Tygodnik powszechny (catholique de gauche). Certains ont écrit pour la revue Kultura, publiée en France pendant toute la période communiste afin de permettre une vie intellectuelle polonaise indépendante.

13. C. Miłosz, Rodzinna Europa, Paris, Instytut Literacki, 1959. En français, l'ouvrage est paru sous le titre Une autre Europe, trad. G. Sédir, Paris, Gallimard, 1964. De la même manière, M. Kundera rappelle la communauté de destin qui lie Europe centrale et occidentale, dans un texte célèbre intitulé "Un Occident kidnappé ou la tragédie de l'Europe centrale», publié dans Le Débat, n ${ }^{\circ} 27,1983$ (5), p. 2-23. 
Occidentaux que le pays dont il vient fait partie de l'Europe. Pour Zbigniew Herbert, comme pour les autres dissidents de sa génération, le sentiment d'appartenance à l'Europe, et le désir d'en faire toujours partie, domine. Le retour sur le passé antique, en particulier dans ses essais intitulés Le Labyrinthe au bord de la mer et Un barbare dans le jardin ${ }^{14}$ s'inscrit, entre autres, dans un projet antitotalitaire : l'Europe occidentale reste pour lui une source de fascination et un recours car elle incarne la continuité organique de toute la culture du Sud, alors que la Pologne a connu la discontinuité sur le plan de la politique comme de la culture.

Il existe néanmoins depuis longtemps une vision critique de l'Europe occidentale en Pologne. L'étonnante Maria Komornicka décrypte dans la culture universitaire de Cambridge les abus du système colonial britannique. Andrzej Bobkowski, très francophile au départ, accomplit un véritable travail de deuil au spectacle d'une France vaincue, occupée et pétainiste. Dans un même ordre d'idées, dans les années 1960, le regard des voyageurs sur l'Italie oscille entre envoûtement et hypercritique, et témoigne de l'ambivalence de l'homme de l'Est face à une Europe occidentale dont il se sent exclu mais dont il constate, non sans un léger sentiment de revanche, la désintégration culturelle. Après 1989, le tropisme vers un Est proche - qu'il est difficile de distinguer de l' "Orient lointain", puisque le polonais désigne les deux par le même mot, Wschód - peut s'inscrire dans le prolongement de cette démystification de l'Occident. C'est le mot Wschód qu'Andrzej Stasiuk a choisi pour titre de l'un de ses derniers livres. En se tournant vers l'Est, comme vers une nouvelle source, certains écrivains critiquent, de façon plus ou moins explicite, l'uniformisation de la planète, livrée au consumérisme. On observe une étrange valorisation de l'Est, voire du retard accumulé pendant l'époque soviétique. S'agit-il d'une nouvelle forme de retour aux sources? D'une volonté de comprendre son passé en explorant les contours d'un univers communiste disparu? De voyager sur les traces d'une histoire déjà perçue comme lointaine bien que proche chronologiquement?

Dans certaines limites, aller vers l'Est, c'est retourner aux sources. La description de l'Ukraine occidentale ou de la Biélorussie, des "confins» [Kresy] et des paysages et des modes de vie caractéristiques, est un véritable lieu commun de la littérature polonaise. Ainsi, le voyage n'est souvent qu'un détour pour revenir à soi. La découverte de territoires proches, aussi bien sur le plan

I4. Z. Herbert, Barbarzyńca w ogrodzie, Varsovie, Czytelnik, I962; en français : Z. Herbert, Un barbare dans le jardin, trad. J. Lajarrige révisée par L. Dyèvre, Paris, Le Bruit du Temps, 20I4; Z. Herbert, Labirynt nad morzem, Varsovie, Zeszyty Literackie, 2000; en français : Z. Herbert, Le Labyrinthe au bord de la mer, traduction et avant-propos B. Gautier, Paris, Le Bruit du Temps, 2011. 
géographique que linguistique et culturel, conduit tout naturellement au constat de la ressemblance. Le Proche est ici le Même. Ceci d'autant plus que plusieurs voyageurs sont dotés d'une double identité qui leur permet d'être à la fois extérieurs et intérieurs au monde qu'ils décrivent : Krzystof Varga est le fils d'un père hongrois et d'une mère polonaise; Henry Shoskhes se meut entre deux identités culturelles, et publie aussi bien en yiddish qu'en polonais. Mais on peut aussi découvrir, chez des voisins proches, des parents habitant pour ainsi dire au coin de la rue, une altérité radicale. C'est le cas pour Henry Shoshkes visitant les shtetlech polonais pendant l'entre-deux-guerres ou, aujourd'hui, pour Mariusz Szczygieł qui explore la culture tchèque afin de mesurer l'abîme qui la sépare de la mentalité polonaise : le catholicisme fervent et la martyrologie des derniers s'opposent diamétralement à l'athéisme viscéral et à la légèreté des premiers. Le retour sur soi peut se produire également lors des voyages vers des terres plus lointaines. Si Bobkowski est déçu par la France d'après «l'étrange défaite», il se montre aussi toujours violemment critique envers ses compatriotes, leur goût de l'héroïsme inutile et du martyr. Dans le cas d'Andrzej Bobkowski, cependant, il est difficile de distinguer les causes des conséquences : il n'est pas exclu qu'il se soit retrouvé en France parce qu'il souhaitait prendre ses distances avec la polonité. Le désir d'un recul critique peut être à l'origine du voyage et non pas seulement advenir au cours de celui-ci.

Les récits de voyage présentés dans ce volume révèlent ainsi une identité en mutation qui remet aussi en cause certains clichés, dont le plus tenace est le poids du catholicisme dans l'histoire, la société et la mentalité polonaises. La place et le traitement que réservent les auteurs abordés dans ce volume à la religion sont remarquables. Dans l'unique récit de pèlerinage de notre corpus, celui de Władysław Reymont, le journaliste athée se sent "étranger chez soi», c'est-à-dire étranger au sentiment religieux des pèlerins, même s'il cède à des moments d'ethnologie participante. L’espérance de la foi semble céder la place à l'angoisse métaphysique qui serait peut-être l'un des traits caractéristiques du voyageur polonais. Dans Dukla, Andrzej Stasiuk écrit : «L’enjeu est de savoir si je vais extraire l'être du non-être ${ }^{15}$." La mort semble plus obsédante en Pologne qu'en Occident : on la médite, on la côtoie. Herbert en Grèce est hanté par la Seconde Guerre mondiale : "Alors que je me trouvais sur l'Acropole, j’invoquais les âmes de mes camarades morts, je déplorais leur sort, [...]. Je répandais des graines de pavot sur leurs tombes oubliées ${ }^{16} »$. Dans Maski

15. Dukla, trad. A. Żuk, L. Alaux, Paris, Christian Bourgois, 2003, p. 202; «Szto o to, czy wywiodę byt z niebytu [...]» (Dukla, Wolowiec, Éditions Czarne, 1997, p. I23).

I6. En français : Z. Herbert, Le Labyrinthe au bord de la mer, ouvr. cité, p. I2I. En polonais : Labirynt nad morzem, ouvr. cité, p. 90 : "[...] stojąc na Akropolu przywotywatem dusze moich polegtych kolegów, użalatem się nad ich losem, [...]. Sypatem ziarna maku na zapomniane groby». 
[Les Masques $]^{17}$, c'est un film sur le carnaval de Venise qui réveille chez l'auteur, Tadeusz Różewicz, l’image de victimes de guerre exhumées : «Les fouilles dans mon pays ont de petites têtes noires / des sourires cruels collés avec du plâtre. » Les Polonais en voyage demeurent hantés par l'Histoire avec sa grande H.

\section{Questions de forme}

Le voyage se raconte, on l'a dit, de différentes manières, mais il inspire deux formes d'écrits diamétralement opposées. D'un côté, le voyage entretient une relation privilégiée avec le reportage, forme a priori liée à l'actualité, obéissant à une déontologie, soucieuse de vérité et d'objectivité, et dont la diffusion repose sur la presse ainsi que, désormais, les nouveaux médias. À l'autre bout, on peut situer le récit de voyage que Dorota Kozicka qualifie d' «intellectuel $^{18}$ ». Dans son récent ouvrage, essentiellement consacré à Zbigniew Herbert, Jarosław Iwaszkiewicz et Jerzy Stępowski, elle interroge cette forme, au croisement du récit de voyage proprement dit, de l'autobiographie et de l'essai. Pourtant, le reportage que les Polonais appellent «littéraire» brouille la limite entre le reportage et le «voyage intellectuel». C’est Ryszard Kapuściński, lauréat de plusieurs prix internationaux, qui a donné à cette forme de reportage ses lettres de noblesse, tout en s'inscrivant - mais à sa manière - dans une tradition déjà existante et illustrée par les reporters Melchior Wańkowicz (I892-1974), Ksawery Pruszyński (1907-1950) et Marian Brandys (1912-1998). Ryszard Kapuściński affiche tout d'abord une déontologie du reporter : refus des itinéraires tracés par les grandes agences, humilité face aux sujets, diversification des angles d'approche. Mais il élabore également une forme à mi-chemin entre l'enquête et la création. Magali Soulatges propose de mettre en relation la forme du reportage selon Kapuściński et la grande vogue, arrivée en Pologne depuis l'Italie avec un certain retard (aux XVII ${ }^{e}$ et XVIII ${ }^{e}$ siècles), des sylvae rerum nobiliaires. Les silvae sont des registres familiaux regroupant des textes indépendants et hétéroclites, qui relèvent de la politique, de l'économie, de la sagesse ou de la chronique, collectionnés sans projet esthétique. Kapuściński se réclame ouvertement de cette forme souple et ouverte, qui tient à la fois du commentaire, de l'essai, du récit, du portrait, de la biographie, de l'anecdote, de la saynète, et qui peut également comprendre cartes et images. Pour Kapuściński, c’est là une

17. T. Różewicz, Poezje zebrane, Wroclaw-Varsovie-Cracovie-Gdansk, Zakład Narodowy im. Ossolińskich, I97I, p. 7.

I8. D. Kozicka, Wędrówcy światów prawdziwych : dwudziestowieczne relacje z podróży [Les Arpenteurs de mondes vrais : les récits de voyage au vingtième siècle], Cracovie, Universitas, 2003. 
manière d'aller à la rencontre d'un monde insaisissable, qui ne peut se raconter "qu'a travers des agencements provisoires de ses différents éclats ${ }^{19}$ ». L'exemple de l'auteur d'Imperium montre qu'entre reportage et "voyage intellectuel» la frontière n'est pas aussi nette qu'on pourrait le penser. Même lorsqu'il se rapproche le plus du témoignage, le reportage ne vise jamais un récit purement factuel. Lorsqu'ils se rendent aujourd'hui, après les faits, sur les théâtres des guerres et des massacres (Bosnie, Moldavie, Rwanda) pour y interroger les témoins, les survivants ou leurs enfants, Andrzej Stasiuk et Wojciech Tochman prennent le temps de comprendre, de reconstituer, de retracer la généalogie des événements. Wojciech Tochman laisse volontiers la parole aux témoins, mais ses livres posent aussi de nombreuses questions à lui-même ou au lecteur, procédé plus proche de l'essai et qui laisse s'exprimer la subjectivité. Le témoignage résonne en nous, suscite des questions qui sont le signe d'une implication personnelle, affective et intellectuelle.

Les récits de voyage ne doivent pas, non plus, être radicalement opposés au roman. Margot Carlier, dans l'avant-propos de son anthologie, met en avant le mélange, dans le reportage de l' "école polonaise», d'un contenu documentaire et de techniques propres au récit de fiction. «Le reporter élabore une véritable narration autour d'un personnage ou d'un événement, et travaille la composition de son texte, en exploitant toutes les possibilités formelles du littéraire ${ }^{20}$.» Il ne s'agit pas là d'une grande originalité, réservée au reportage polonais. Avec De sang-froid, Truman Capote a déjà magistralement montré comment les techniques romanesques pouvaient être mises au service de la vérité et donner lieu à un roman-vérité, ou non-fiction novel. D’ailleurs, l'évolution simultanée du reportage et du roman a déjà été largement remarquée dans différentes aires culturelles et linguistiques ${ }^{21}$. Cependant, dans le cas polonais, Margot Carlier propose d'expliquer le travail de la forme par la nécessité de déjouer la censure à l'époque communiste :

On dit souvent en Pologne que la qualité littéraire de sa littérature documentaire doit beaucoup à la censure communiste. [...] Il n'y avait pas d'autre moyen de traiter du collectif que de décrire le particulier. [...] Cela a engendré un récit décalé, appelé alors «à double fond"; un récit subtil, à la thématique en apparence banale, mais où le détail dépassait le sens premier, se trouvant doté d'une signification

19. M. Soulatges, "Le modèle de la silva rerum dans l'écriture du grand reportage par Ryszard Kapuścinski», Anabases [Online], Io | 2009, <http://anabases.revues.org/728> [consulté le 22/03/2016].

20. M. Carlier (éd.), La vie est un reportage. Anthologie du reportage polonais, Montricher, Noir sur Blanc, 2005, p. II.

2I. M. Boucharenc (dir.), Roman et reportage. Rencontres croisées, Limoges, PULIM, 2015. 
métaphorique, plus large, plus universelle. Nous disions du reportage qu’il était l'art de voir la mer dans une goutte d'eau, écrivait Adam Michnik²2.

Dans un même ordre d'idées, Przemysław Czapliński parle à propos de la Pologne populaire, d'une situation «proprement orwelienne ${ }^{23}$ », qui consiste en un "blocage de l'évidence» [blokada oczywistości]. Dans un texte rédigé pour le premier numéro de la (bientôt célèbre) revue clandestine Zapis ${ }^{24}$, publié en 1977, le poète Stanisław Barańczak explique la signification du titre de la revue : le mot Zapis fait référence à la censure - suivi d'un numéro, il est apposé sur les livres mis à l'index - mais ce terme signifie également "notation" et traduit ainsi la conviction des rédacteurs que l'observation et la notation, c'est-à-dire la fixation en mots de tout ce qui a pour eux valeur de vérité, n'est pas seulement le droit de l'auteur mais son devoir. Comment les techniques propres à la fiction contribuent-elles à mettre en valeur le vrai? Quelle que soit la réponse, la fin du communisme en 1989 n’a pas détourné les auteurs du reportage littéraire polonais de leur ambition de témoigner, ni de leur ambition esthétique. Mariusz Szczygieł semble pratiquer l'écriture à contraintes, quand il explique dans un entretien qu' " un bon reportage fonctionne comme un bon roman": il s'agit de trouver la première et la dernière phrase et de les "relier par une dramaturgie 25 ».

D'autres traits d'écriture témoignent de fortes interactions entre sciences sociales et reportage, ou littérature et savoir. L'influence des sciences n'est pas moindre dans les voyages de proximité que dans les voyages lointains qui mobilisent, presque nécessairement, histoire, géographie, ethnologie et anthropologie. Les reportages d'Henry Shoshkes s'inscrivent dans le contexte de l'ethnologie juive moderne qu'ont forgée les folkloristes, comme Shalom Anski, en Russie. La prose d'Olga Tokarczuk fait de toute évidence écho aux travaux du sociologue Zygmunt Baumann, auteur de La Société liquide. Dans Mordre dans la pierre, Wojciech Tochman raconte comment, dans la Bosnie d'après-guerre, un médecin reconstitue les squelettes pour que les familles puissent donner une sépulture à leurs morts et faire enfin leur travail de deuil. Le reporter accomplit un tour de force grâce au transfert vers le reportage d'un lexique scientifique (archéologie, paléontologie, médecine légale), avec un violent effet d'anachronisme, lorsqu'un vocabulaire habituellement utilisé

22. M. Carlier, La vie est un reportage. Anthologie du reportage polonais, ouvr. cité, p. II.

23. P. Czapliński, Powrót Centrali, Cracovie, Wydawnictwo Literackie, 2007, p. I54-I55.

24. "Dlaczego zapis», Zapis, n I, I977, cité par P. Czapliński, dans Powrót Centrali, p. I54.

25. "Eine gute Reportage funktioniert wie ein Roman», entretien du 20 avril 2006 avec V. Hummel pour le journal en ligne Netzzeitung, <https://web.archive.org/web/20060425044345/> <http://www.netzeitung.de/voiceofgermany/393320.html> [consulté le 23/05/2015]. Cité d'après M.-O. Thirouin, ci-dessous. 
dans l'étude des périodes anciennes est appliqué à des morts récents, victimes de la guerre de Yougoslavie dans les années 1990. Les vocables scientifiques remplacent les procédés traditionnels du pathos, mais leur fausse froideur provoque un choc au moins aussi fort.

L'avant-dernier article de ce volume, rédigé par une chercheuse en sciences de l'information et de la communication, invite à nuancer la vision d'une Pologne "citoyenne du monde». L'article étudie le traitement de la question de l'immigration dans quatre hebdomadaires polonais entre 2012 et 20I4. Il en ressort que la Pologne, pays anciennement multiculturel, devenu ethniquement homogène sous le communisme, pays d'émigration depuis plus de deux siècles, refoule désormais une partie de son identité et de son expérience historique. Les élections parlementaires qui ont eu lieu en Pologne, le 25 octobre 2015, deux jours après la fin du colloque ont fait écho aux résultats de cette analyse.

Il y aurait donc un écart entre la représentation littéraire et la réalité? $\mathrm{La}$ divergence entre la littérature du voyage dont traite le présent volume et la presse polonaise reflète sans doute, d'abord, le clivage entre les élites et les masses. Mais il est possible d'interpréter le contraste autrement : la difficulté de la société polonaise à affronter la xénophobie et le racisme ne serait-elle pas liée au fait que le phénomène migratoire est traité dans des articles courts, factuels, et non dans des reportages longs, subjectifs, qui permettent d'humaniser l'Autre, et de le percevoir comme notre semblable? Le reportage pourra-t-il contribuer à changer le visage actuel de la Pologne et aider celle-ci à renouer avec sa tradition d'humanisme et d'universalisme, si bien incarnée par Maria Komornicka qui disait : «je suis humain, ma patrie, c'est le monde ${ }^{26}$ »?

26. "[...] ojczyzna moja świat, jestem cztowiekiem» (M. Komornicka, Raj mtodzieży. Wspomnienia z Cambridge [Le Paradis de la jeunesse. Souvenirs de Cambridge], Przeglad pedagogiczny [Revue pédagogique], nº 5-7, 1896, p. 98. Cité d'après M. Chmurski, ci-dessous. 